\title{
Competitive Spectrum Sharing in Wireless Networks: A Dynamic Non-cooperative Game Approach
}

\author{
Omar Raoof, Zaineb Al-Banna, and H.S. Al-Raweshidy \\ Brunel University, Wireless Networks and Communications Centre (WNCC), \\ London, UB8 3PH, UK \\ \{Omar.Raoof, Zaineb.Al-Banna\} @brunel.ac.uk
}

\begin{abstract}
Game Theory" is a promising mathematical tool to improve the utilization of radio frequency spectrum in wireless networks. In this paper, we consider the problem of spectrum sharing between a primary user and a group of secondary users. We formulate our solution in such a way that one of the secondary users will be a secondary primary user by sharing the spectrum offered from the primary user and offer his share to be shared by the rest of the secondary users in the network. A theoretical non-cooperative game model is introduced to study node behavior in wireless networks based on their reputation. The only way for a node to guarantee a share in the spectrum is by enhancing its reputation, which is done by serving other nodes in the network. Game theory can be used by individual selfish nodes to determine their optimal strategy for participation level in the network. Furthermore, game theory produces information about the overall nature of nodes' interaction and system efficiency, showing how system efficiency can be improved.
\end{abstract}

Keywords: Spectrum sharing, game theory, spectrum pooling, non-cooperative game.

\section{Introduction}

Today's wireless communication occupies the bulk of the research in the field of communications because of the increasing demand of wireless services. It has been predicted that in the next five years, about $55 \%$ of all users will access the Internet wirelessly [1] which leads to the need for more efficient use of frequency, where the scarce resources require wisdom of distribution and utilization. Cognitive technique is considered a candidate for the optimum use of the frequency spectrum.

Spectrum users can be classified to be Primary users (PU) and Secondary users (SU) (i.e. licensed and unlicensed users respectively). However the licensed frequency is not used completely by the primary users there are holes left in the licensed band. In a cognitive radio system, secondary users seek for these holes and opportunistically use them. The benefit then returns to both primary user which can get revenue from selling its own spectrum, and secondary users by subleasing available channels. In [2] the author mentioned that Neel and Reed have studied how to apply game theory to a radio as a member of a cognitive network. With game theory they were able to analyze protocols to determine whether cognitive radio behaviour 
could result in a stable network behaviour, and they conclude that it can. Generally, the PU's are the license holders and the SU are allowed to use frequency band when the PU are inactive however they are required to vacate when the PU are active again. The arrival of PU causes the SU to lose that band and therefore link maintenance become a key issue. Spectrum pooling (shown in figure 1) is one of the solutions to this problem, where the entire band is divided into large number of sub-channels in such a way that they lie in a different PU's bands. This solution minimizes the chance of losing the entire sub-channel upon the arrival of the PU, as no PU can cause the complete breakdown of the SU link.

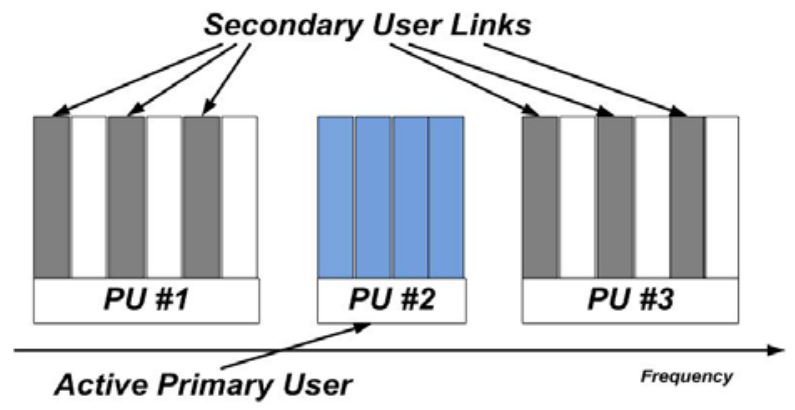

Fig. 1. Spectrum Pooling

In this paper we used game theory to model the utilization of the spaces and holes which were found in the licensed band in a competitive manner among multi secondary users, as shown in figure 1. It is assumed that the search and detection of vacancies within the licensed spectrum is already carried out by implementing the methods of spectrum sensing. Then secondary users will compete to share the spectrum frequency with the primary user according to a pricing function, which is computed by the primary user's administrator. It also depends on the dimensions similar to those which are considered in assessment of the frequency license as [2]:

a) Bandwidth required (in $\mathrm{MHz}$ ).

b) Demand within the service region.

c) Duration of the contract.

d) Opportunity for growth of services within the service region.

e) Cost of installing and providing service.

The cost then has been assumed as units with five categories. Each SU takes one of these five categories and will compete with the other SU according to number of units it obtained. The first priority will be granted to the SU which has maximum number of units and assigned the first category and second priority will be granted to the SU which collected maximum units of all the rest and so on.

The rest of the paper is organized as follows; the following part of this paper presents some related work to this area, giving brief details of what have been achieved as well. Following from this, section ' 3 ' explains how to use game theory as a tool to predict and strategies node behaviour in wireless communications. It presents a game theoretical model that controls how SUs' access the shared spectrum. Nash equilibrium 
strategy is defined in section '4', and properties of this strategy are discussed in section ' 5 '. Finally, conclusion and proposed future work are presented in the last section.

\section{Related Works}

An introduction to cognitive radio was provided in [3] where the fundamental cognitive tasks as well as the emergent behavior of cognitive radio were discussed. The problem of spectrum pricing in a cognitive radio network where multiple PU's providers compete with each other to offer spectrum access to SU's is presented in [4] and [5]. By using equilibrium pricing scheme, each of the primary service providers aims to maximize its profit under quality of service (QoS) constraint for PU's. The authors adopted a utility function to obtain the demand function. The stability of the proposed dynamic game algorithms in terms of convergence to the Nash equilibrium is studied. However, Nash equilibrium is not efficient in the scene that the total profit of the primary service providers is not maximized. Finally, PU's may deviate from the optimal solution reached by the Nash equilibrium stage; a punishment mechanism may be applied to the deviating primary service providers.

In [6] the problem of the spectrum sharing between the PU and SU were considered. The authors formulate the named problem as an oligopoly market competition and use a non-cooperative game to obtain the spectrum allocation for SU's. Numerically the authors investigated the stability conditions of the dynamic behavior for the spectrum sharing scheme.

\section{Game Theory as a Tool to Predict and Strategize Spectrum Usage}

In this section a theoretical game model is presented for the case where all the SU's can completely observe the strategies and the payoff of other SU's. Mainly, the SU's are fighting with each other in order to get to share the spectrum with the PU (i.e. the players in the game are the secondary users); here we assume that one of the SU's will try to be a secondary PU (i.e. the $S U$ will become responsible of the offered spectrum by the PU and will decide whether to share it with the other SU's by pooling the offered spectrum again or not). In this case the SU will use the spectrum that the PU is offering and shares it with the other SU's simultaneously. This mechanism will save time and assures that all the SU will receive a fair share of the spectrum. A node with low priority traffic will not be interested in paying more money to share the spectrum with the PU and compete with the other SU's rather than sharing it with the $\mathrm{SU}$, and the opposite works for the node with high priority traffic.

Game theory mostly attractive to analyze the performance of all kind of problems related to wireless networks. The reason is that it has the ability to model individual, independent decision makers whose actions potentially affect all other decision makers. Furthermore, game theory is a field of applied mathematics that describes and analyzes interactive decision situations. It consists of a set of analytical tools that predict the outcome of complex interactions among rational entities, where rationality demands a strict adherence to a strategy based on perceived or measured results [7]. 
Game theory studies strategic interaction in competitive, non-cooperative, and cooperative environments. Half a century old, it has already revolutionized economics, and is spreading rapidly to a wide variety of fields. In the early to mid 1990's, game theory was applied to networking problems including flow control, congestion control, routing and pricing of Internet services [7]. Non-cooperative and Cooperative games were introduced by John Nash (in papers between 1950- 1953), his most contribution was his existence proof of an equilibrium state in non-cooperative games, the Nash equilibrium [7]. More recently, there has been growing interest in adopting game-theoretic methods to model today's leading communications and networking issues, including power control and resource sharing in wireless and peer-to-peer networks. Moreover, leading computer scientists are often invited to speak at major game theory conferences, such as the World Congress on Game Theory 2000, 2004 and 2008 [8]. So far, evidence of previous researches shows that game theory may be an appropriate tool to solve some problems in communications systems; this section presents some of the most important concepts of game theory and some particular concepts which is very important in our proposal. Normally, any game $G$ has three components: a set of players, a set of possible actions for each player, and a set of utility functions mapping action profiles into the real numbers. In this paper, the set of players are denoted as $I$, where $I$, is finite with, $i=\{1,2,3, \ldots \ldots \ldots, I\}\}$. For each player $i \in \mathrm{I}$ is denoted by $A_{i}$ the set of possible actions that player $i$ can take, and $A$, which is denoted as the space of all action profiles is equal to:

$$
A=A 1 \times A 2 \times A 3 \times \ldots \times A I .
$$

Finally, for each $i \in \mathrm{I}$, we have $U_{i}: A \rightarrow R$, which denotes $i$ 's player utility function. Another notation to be defined before carrying on; suppose that $a \in A$ is a strategy profile and $i \in I$ is a player; and then $a i \in A i$ denote player $i$ 's action in $a$ and $a-i$ denote the actions of the other $I-1$ plyers.

The most important equilibrium concept in game theory is the concept of Nash Equilibrium [7]. A Nash equilibrium is an action profile at which no user may gain by unilaterally deviating. So a Nash equilibrium is a stable operating point because no user has any incentive to change strategy. More formally, a Nash equilibrium is a strategy profile $a$ such that for all $a i \in A i$,

$$
U(a i, a-i) \geq U(\tilde{a} i, a-i) .
$$

The ã, denote another action for the player $i$ 's.

Pareto efficiency is another important concept for our application of game theory. An action profile $a \in A$ is said to be Pareto if there is no action profile $\tilde{a} \in A$ such that for all $i$,

$$
U(a i) \geq U(\tilde{a} i) .
$$

In another word, an action profile is said to be Pareto efficient if and only if it is impossible to improve the utility of any player without harming another player.

In this paper, we assume that the network lifetime is infinitely long and divided into individual time periods, represented by $t$ for each $t=0,1,2, \ldots \ldots, \infty$. At each time period $t$ a node sends a request for share in the spectrum from the PU. Other activities that each node follows are to share its requirement of the spectrum with the 
other nodes. By this the node will decide whether it's worth it to share the spectrum with the PU and become a secondary PU, or give a chance to the other nodes to do so.

In this paper we will represent the infinitely repeated version of game $G$ (i.e. this is the case when $G$ is going to be played over and over again in successive time periods) by $G^{\infty}$. In this paper we are assuming that the PU is offering a single frequency band to be shared by other SU's. However, if the PU is planning to offer more bands then the proposed mechanism must be repeated for the other bands between the secondary users.

We will define the node reputation as $R$ which will depends on the node performance during any time period $t$ as well as in prior time periods. Reputation of player $i$ in some time period $t$ is denoted by $R_{t}^{i}$. Formally, we define node reputation as follows:

$$
R_{t}^{i}=R_{t-1}^{i}(1-\propto)+w \times \propto \quad 0 \leq \propto \leq 1, t \geq 2 .
$$

Where the following " $\propto$ " is the history of the node, it depends on the node reputation in the previous time periods according to node movements. The " $w$ " is equal to " 1 " when player $i$ at time $t$ is interested in sharing the offered spectrum and " 0 ". Therefore, $0 \leq R_{t}^{i} \leq 1$, i.e. the reputation of each player is a value form " 0 " and " 1 " (including) $\left(R_{t}^{i} \in[0,1]\right)$. Moreover, the reputation value of all players is equal to " 0 " when $t=0$. A high value of $\propto$ means the more importance is assigned to a player's need to share the spectrum with the PU (higher priority) in the current time period than its previous need record, and vice versa. Thus, when $\propto$ is high, a node with even low reputation value in the current time period $t$, can significantly improve its reputation when it realises that it needs a better share of the spectrum.

\section{Nash Equilibrium of the Repeated Game $\mathbf{G}^{\infty}$}

As was defined the Nash equilibrium case earlier, the evaluation of the Nash equilibrium of the repeated game $G^{\infty}$ will be engaged. By finding the Nash equilibrium of $G^{\infty}$ it leads to the deduction of the Nash equilibria of $G$. The proposed incentive mechanism is based on a player's reputation $R$ links, the benefit of which is that a player draws from the system to its contribution the benefit is a monotonically increasing function of a player's contribution. Thus, this is a non-cooperative game among the players, where each player with high priority traffic wants to maximize its utility. The classical concept of Nash equilibrium points a way out of the endless cycle of speculation and counter-speculation as to what strategies the players should use. The intent is to deduce a symmetric Nash equilibrium because all the players belong to the same population/network (i.e., assume the same role) and it is therefore easier (i.e., require no coordination among players) to achieve such an equilibrium. If the players in a game either do not differ significantly or are not aware of any differences among themselves (i.e., if they are drawn from a single homogeneous population) then it is difficult for them to coordinate, and a symmetric equilibrium, in which every player uses the same strategy, is more compelling.

The argument of a single homogeneous population implies that all the peers in a wireless mesh network have equivalent responsibilities and capabilities as everybody else. We assume that if the player chooses the action \{want to share $\}$ to the access 
point, this will assign him a probability of $p$, and if the player chooses the action \{does not want to share\}, this will assign one a probability of $1-p$.

It must be mentioned that in the action profile, all the players choose the action \{does not want to share\}; this is a time and money saving Nash equilibrium case. As this will mean that the nodes are not interested in sharing the spectrum for the entire communication time at all. That is to say nodes have low priority traffic and accessing the spectrum will be by chance, nodes will not compete to send their data and will not offer more money to the PU to get the spectrum. If any other player $i$ decided to switch to the action $\{$ want to share $\}$ instead, its payoff will be $-C$ which is less than a payoff of " 0 " that the node gets when decide not to share the spectrum. If all the players choose the action \{want to share\}, this is an undesirable Nash equilibrium case. This is easy to see because all the nodes will have to compete against each other again, this will waste time and the winner will be the PU, as one of the SU's should offer more to share the offered spectrum.

The expected payoff of any player in time period $t$ when it selects the action \{want to share \} is:

$$
p\left(-C+R_{t}^{\text {share }} \times U\right) .
$$

This payoff is denoted as Payoff $f_{\text {share }}$. Similarly, the payoff for any player selects the action \{does not want to share $\}$ will be:

$$
(1-p)\left(R_{t}^{\text {don'tshare }} \times U\right) .
$$

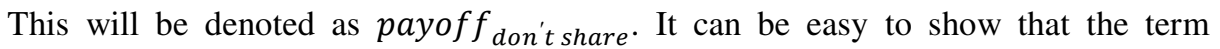
$R_{t}^{\text {share }} \times U$ captures the notation that the probability of SU becoming a secondary PU by sharing the offered spectrum is directly proportional to node's reputation.

$R_{t}^{\text {share }}$ is player reputation when it wants to share the offered spectrum at time $t$ (i.e. $w=1$ in equation 4 ), and $R_{t}^{\text {don'tshare }}$ is player $i$ reputation when it decides to take the action $\{$ does not want to share $\}$ at the same time period $t$ (i.e. $w=0$ in equation 4), from equation 4 , we can get:

$$
R_{t}^{\text {share }}=R_{t-1}(1-\propto)+\propto
$$

and

$$
R_{t}^{\text {don'tshare }}=R_{t-1}(1-\propto) .
$$

Generally, each player's expected payoff in equilibrium is its expected payoff to any of its actions that it uses with positive probability. The above useful characterization of mixed-strategy Nash equilibrium yields to;

$$
\text { payof } f_{\text {share }}=\text { payoff } f_{\text {don'tshare }} .
$$

Using equations 5, 6, and 7;

$$
p\left(-C+\left(R_{t-1}(1-\propto)+\propto\right) \times U\right)=(1-p)\left(R_{t-1}(1-\propto) \times U\right) .
$$


Solving equation 9 to get the final value of $p$;

$$
p=\frac{R_{t-1} \times U \times(1-\propto)}{-C+2 R_{t-1} \times U \times(1-\propto)+U \times \propto} .
$$

It must be mentioned that the value $p$ obtained above is not a constant, but varies in each time interval depending upon a node's reputation at the end of the previous time interval $t-1$.

Finally, the mixed strategy pair $(p, 1-p)$ for actions \{want to share, does not want to share respectively, is a mixed strategy Nash equilibrium for the players (i.e. nodes in the network). Assuming no collusion among nodes, if all the other nodes follow the above strategy, then the best strategy for any node is to also follow the above strategy. Actually, this is a symmetric mixed strategy Nash equilibrium for any $G$, as well as $G^{\infty}$. In fact, it is a more stable equilibrium than the one in which no node is interested in sharing the offered spectrum. This is due to two reasons. First, when none of the nodes is interested in sharing the spectrum, the network is not useful to any user. Second, in practice nodes, which derive finite utility from altruism, would always send some messages irrespective of how much they obtain in return. Therefore, it is unlikely to have a scenario in which no node is looking to contact the PU to share the spectrum.

\section{Properties of the Proposed Nash Equilibrium}

In this section, we will present some of the interesting properties of the Nash equilibrium derived in the section above (equation 10).

\subsection{Simplicity of Calculating the Nash Equilibrium}

In section ' 4 ' we have calculated the probability of achieving the equilibrium point between the nodes based on which node will decide to share the spectrum with the PU and become a secondary PU. In each play of the game (or time period $t$ ), players (wireless nodes) based on their reputation at the end of the prior time period decide whether they should ask to share the offered spectrum or not. This probability as one can see does not remain constant from one period to another, and depends on a player's reputation at the end of the last time period. Players can calculate their reputation using equation 4 , since they know precisely their actions at each play of the game. Thus, determining the Nash equilibrium strategy is fairly straightforward for any player. However, it must be noted that there is an inherent assumption that nodes get serviced based on their current reputation.

Figure 2, shows how players' reputations change in every time interval depending on their Nash strategy. At the beginning of the communication time, both, player 1 and 2 are competing with each other to guarantee access to the offered spectrum. However, player 1 uses the spectrum but at the same time managed to help player 2 (i.e. player 1 will be the secondary PU and will manage the access of players 2 and 3 to the offered spectrum; player2 will have a better chance as it has a better reputation). Player 3 shows his interest in the offered spectrum after the third time interval, and managed to use the spectrum once both player 1 and 2 finished using it or they are not interested anymore in sharing it. The figure shows the players (nodes) reputation values $0 \leq R_{t}^{i} \leq 1$ over ten time intervals. 


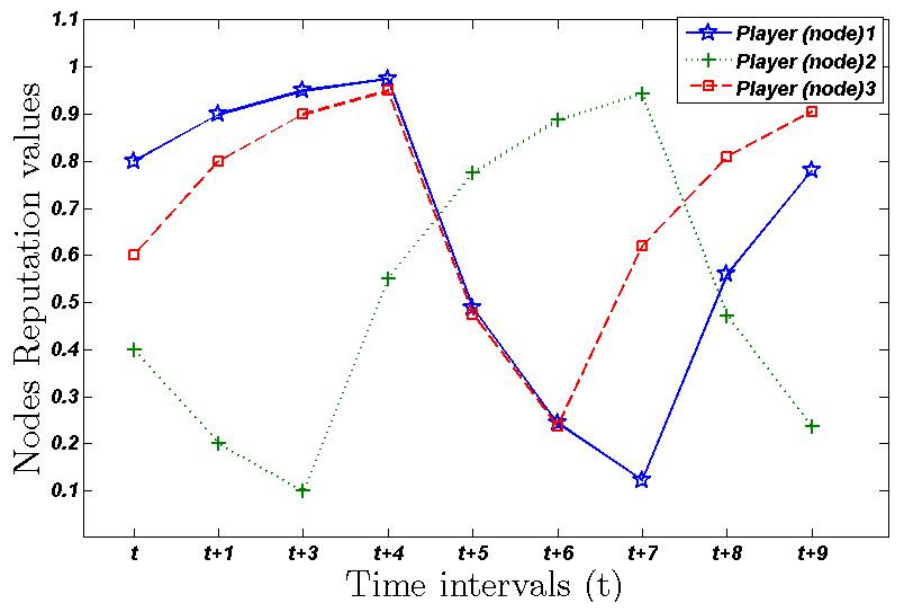

Fig. 2. Change in player's reputation controlled by their Nash equilibrium strategies

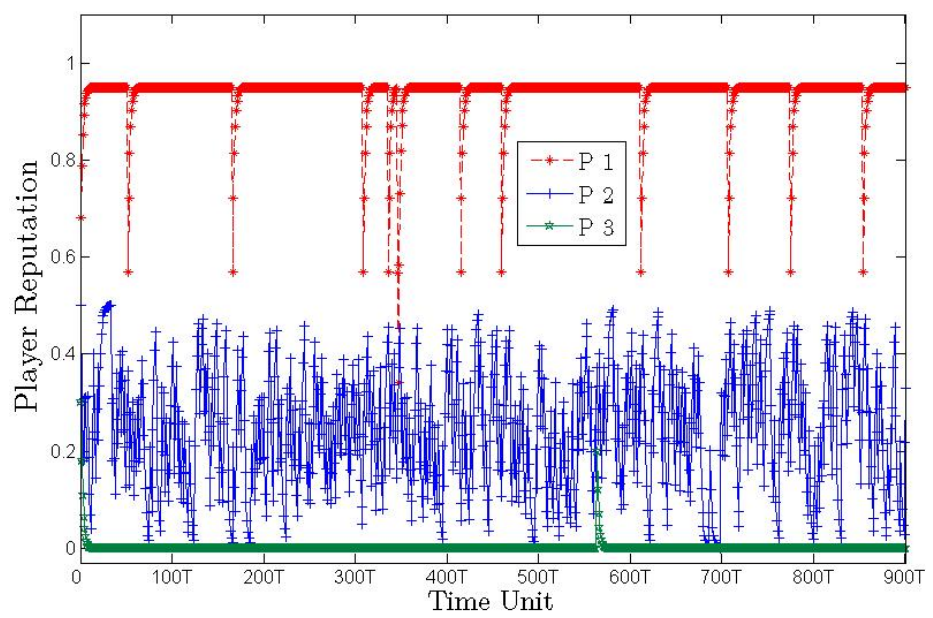

Fig. 3. Changing player reputation over a longer time period

On the other hand, figure 3 above shows the same result but over a longer time period, around nine hundred time intervals. Similarly, three nodes are competing with each other, with player one with highest reputation and player three with the lowest. Player 1 will act as the secondary PU over the other two users (i.e. player 2 and 3). In this figure we used a random matrix generator to show different reputation when player 1 is interested to share the spectrum for $80 \%$ of the time, player 2 for $50 \%$ of the time and player 3 for $8 \%$ of the time only.

\subsection{Addressing the Spectrum to the Right Node Faster}

The simple game theoretic model presented in this paper, wherein node reputation is used as a basis for deciding who will share the offered spectrum, predicts that it is in 
every peer's best interest to serve others. This includes the nodes that are not interested to share the spectrum at the current time period. Our simulations support this behavior as we found that the total service received by a node is balanced by the total service that it has to offer to others, as shown in Figure 2.

\subsection{Addressing the Problem of Competitive Sharing}

An important property of the equilibrium emerges from equation 10 that predicts the probability with which one node will be a secondary PU and it should serve others. If we set the value of $C$ in away such that, $C<<<U$ (i.e. $C$ can be ignored from equation 10$)$, then equation 10 would be;

$$
p=\frac{R_{t-1}(1-\propto)}{2 R_{t-1}(1-\alpha)+\propto} .
$$

That would lead us to the conclusion that $p<0.5$, hence showing that if the cost of providing a service to other nodes (i.e. sharing the shared spectrum), Nash equilibrium of the proposed game predicts that players should help each other less than 50 percent of the time when the spectrum is offered by the PU. This, although it appears to be very restrictive, is a consequence of the fact that all nodes are selfish and are better off trying to share the spectrum than serving others. Intuitively, if a node knows that everyone else in the network behaves selfishly, i.e., provide as little service as possible, then the best strategy for the named node cannot be to serve others most of the time (i.e., with probability greater than 0.5 ).

\subsection{Fairness, and Equal Sharing of Cost and Spectrum}

We concluded from the previous section that serving with a priority of less than 50 percent (i.e. when $C<<<U$ ) is an optimal point, the observer can notice that the overall system efficiency is severely reduced. This is because most of the nodes in the network act selfishly and at least half of the service requests from other nodes will are not fulfilled. On the other hand, this equilibrium strategy provides fairness in the sense that the cost of system inefficiency is not burn by a single node (i.e. has one positive side), but it is shared among all nodes. This is because each node's request is likely to be turned down by the serving node (i.e. selfish secondary PU). In this paper, we assume that if a node's request at one node is turned down, the node tries at some other candidate node capable of serving the request. On average, the probability that a node's request is successfully served in a time period is proportional to its current reputation.

\subsection{Decreasing $\propto$ for a Better Share of the Spectrum}

Figure 4 shows the effects of $\propto$ on the reputation probability of the nodes in the case where the node is not interested in sharing the spectrum. On the other hand, the node in figure 5 is looking to keep its share of the spectrum (derived from equation 7). 


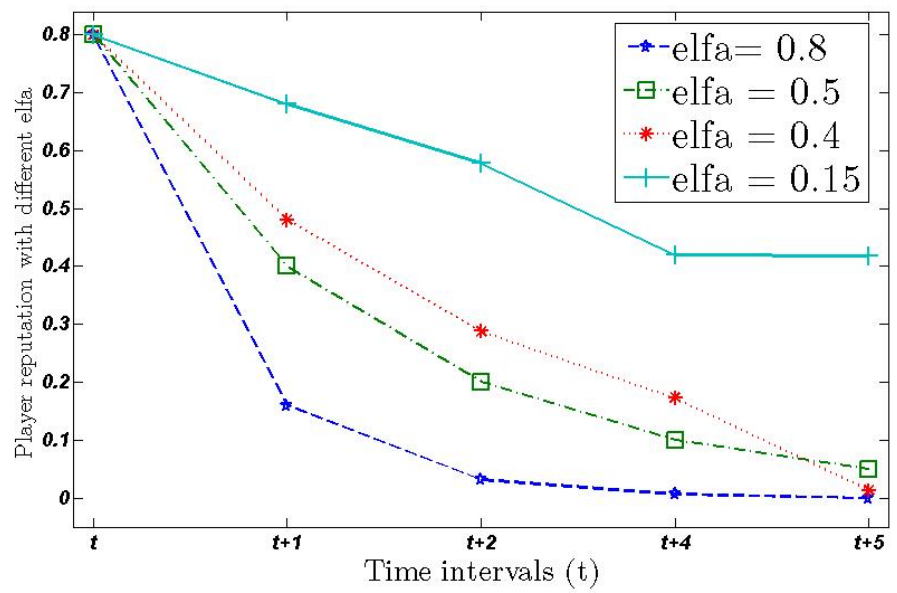

Fig. 4. Players reputation with respect to $\propto$, and the node is not interested in sharing the offered spectrum

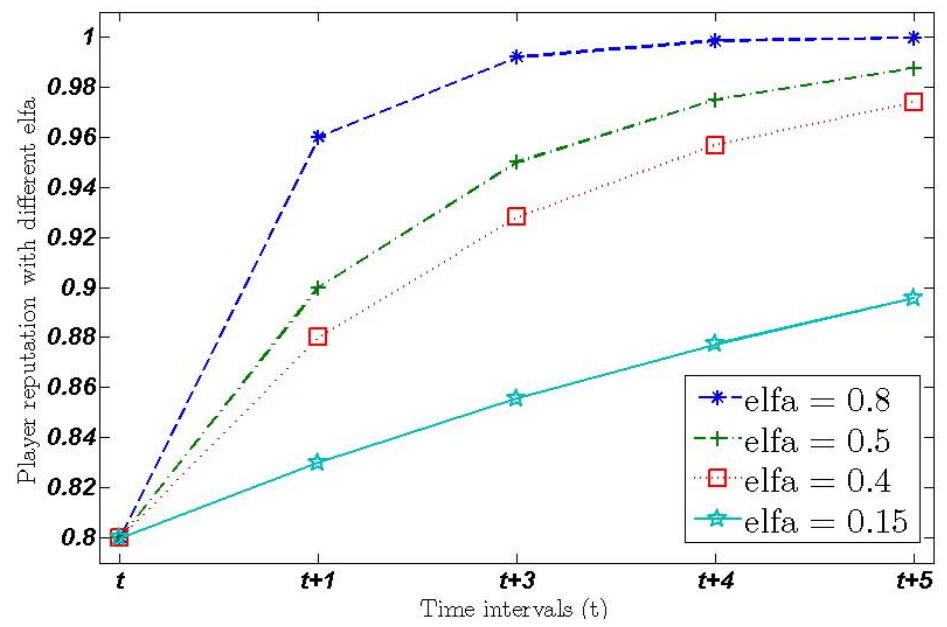

Fig. 5. Players reputation with respect to $\propto$, and the node is definitely interested in sharing the offered spectrum from the PU

As can be seen from figure 4 and 5, a lower value of $\alpha$ shifts the reputation probability curve upwards. However, that all depends on whether the node is interested in using the offered spectrum or not. If the node is looking to give its share of the spectrum to the other nodes, a low value of $\propto$ will gradually help the node to lose its share, however a high value of $\propto$ will guarantee a faster release of the spectrum. This is true for figure 5 as well, which is to be expected since $\propto$ determines how much importance is given to a node's current performance as compared to its past service record. A low value of $\propto$ (i.e., giving more importance to nodes past actions up to the current time period $t$ ) means that nodes need to continually provide service to be able to maintain high reputation and access spectrum offered from the PU. If however $\propto$ is high, nodes 
can easily increase their reputation in any period in which they provide service to other nodes. This is irrespective of how cooperative they have been in the past with regards to providing service to others. Therefore a simple way to improve the system efficiency is to set $\propto$ as low as possible.

\section{Conclusion and Future Work}

In this paper, we have proposed a simple non-cooperative game mechanism based on a node's reputation to overcome the competitive spectrum sharing problem prevalent in wireless networks. The model presented here addresses the spectrum sharing problem as it predicts that even for selfish users serving others is the best strategy. Game theory is used to predict the optimum (Nash equilibrium) strategies of selfish nodes such that their profits are maximized. Game theory is also used to provide valuable insight into the behavior of individual nodes, as well as the performance of the overall system. Interestingly, game theory provided us proof for some of the intuitive results, such as the strategy of serving less than 50 percent of the times when the cost of providing service is negligible. The proposed game theoretic solution of the spectrum sharing problem has several significant advantages; fairness, simple implementation, and ease of calculating optimum strategies. For simplicity we assumed that the cost and utility attached with serving and obtaining services is the same for all service types and for all users. In future, developing more elaborate models that take into account the heterogeneity of service types and users would be desired. Also, we would investigate the applicability of game theory in cognitive radio systems when heterogeneity is the overriding factor in the designing of protocols for system operations.

\section{References}

1. Nagaraj, S.V.: Entropy-based spectrum sensing in cognitive radio. San Diego State University, 5500 Campanile Dr., San Diego (2008)

2. Arslan, H. (ed.): Cognitive Radio, Software Defined Radio, and Adaptive Wireless Systems, University of South Florida, Tampa. Springer, Heidelberg (2007)

3. Haykin, S.: Cognitive radio: Brain-empowered wireless communications. IEEE J. Select. Areas Comm. 23(2), 201-220 (2005)

4. Niyato, D., Hossain, E.: Competitive Spectrum Sharing in Cognitive Radio Networks: A Dynamic Game Approach. IEEE Transactions on Wireless Communications 7(7), 26512660 (2008)

5. Niyato, D., Hossain, E.: Equilibrium and Disequilibrium Pricing for Spectrum Trading in Cognitive Radio: A Control-Theoretic Approach. In: IEEE GLOBECOM (2007)

6. Niyato, D., Hossain, E.: Competitive Pricing for Spectrum Sharing in Cognitive Radio Networks: Dynamic Game, Inefficiency of Nash Equilibrium, and Collusion. IEEE Journal on Selected Areas in Communications 26(1), 192-202 (2008)

7. MacKenzie, A.B., DaSilva, L.A.: Game Theory for Wireless Engineering, 1st edn. (2006)

8. Games 2008, Third World Congress of the Game Theory Society, http: / /www. kellogg.northwestern. edu/meds / games2 008 / 